\title{
BMJ Open Quality Journey to zero serious bacterial prosthetic joint infection
}

\author{
Elisabeth Duvaland, ${ }^{1}$ Steen Villumsen, ${ }^{2}$ Mette Walberg, ${ }^{3}$ Vibeke Hanch-Hansen, ${ }^{4}$ \\ Marianne Olsson, ${ }^{1}$ Lukas Maansson (i) ${ }^{1}$
}

To cite: Duvaland $\mathrm{E}$, Villumsen S, Walberg M, et al. Journey to zero serious bacterial prosthetic joint infection. BMJ Open Quality 2021;10:e01134. doi:10.1136/ bmjoq-2020-001134

Received 30 July 2020 Accepted 15 October 2021

Check for updates

(C) Author(s) (or their employer(s)) 2021. Re-use permitted under CC BY-NC. No commercial re-use. See rights and permissions. Published by BMJ.

${ }^{1}$ Orthopedic Surgery and Emergency Medicine, Vestre Viken Hospital Trust, Drammen, Norway

${ }^{2}$ Internal Medicine, Vestre Viken Hospital Trust, Drammen, Norway

${ }^{3}$ Infection Prevention, Vestre Viken Hospital Trust, Drammen, Norway

${ }^{4}$ Orthopedic Surgery and Emergency Medicine, Vestre Viken Hospital Trust, Kongsberg, Norway

Correspondence to

Dr Lukas Maansson;

lukas.mansson@vestreviken.no

\section{INTRODUCTION}

Deep infections following primary joint replacement cause great suffering to patients and have high treatment costs. ${ }^{1}$ In the general population, and especially in those that have recent contact with healthcare facilities, the bacteria Staphylococcus aureus often colonise the skin and the upper airways without causing disease. However, indolent in the skin, the same bacteria may cause devastating infection associated with implant devices and not least in orthopaedic surgery.

There is extensive literature on specific measures aimed at reducing patients' own bioburden with $S$. aureus before surgery summarised in the National Institute of Health and Care guidelines. ${ }^{2}{ }^{3}$ Universal strategy is more cost-effective than a 'screen and treat' strategy. ${ }^{2}$ Use of triclosan-coated sutures is recommended by several major public health organisations. ${ }^{245}$

In contrast, we found one single publication describing the effect of prolonged decolonisation on the incidence of prosthetic joint infection (PJI) after surgery. ${ }^{6}$ However, there has been more attention towards postoperative factors on PJI. ${ }^{7}$

In an overall 'zero harm' healthcare improvement project at Drammen Hospital (DH) and Kongsberg Hospital $(\mathrm{KH})$, part of the Vestre Viken Hospital Trust, we aimed to eliminate the incidence of PJI caused by $S$. aureus. Compliance with the decolonising protocol is a major issue for success. Hence, in this quality improvement project, we developed a highly standardised protocol for all patients admitted for acute (hip only) and planned surgeries (hip, knee, shoulder). The combined intervention included use of triclosan-coated sutures and patient decolonisation on the incidence of PJI caused by $S$. aureus. We are not aware of any similar previous reports.

\section{METHODS}

Patients admitted for primary joint replacement surgery (shoulder, knee or hip surgery) were included in our project. Three PlanDo-Study-Act (PDSA) cycles were used to stepwise introduce our quality improvement combined intervention. We developed a standard operating protocol that aimed to secure adherence and standardisation of the intervention through PDSA cycles with the doctors and nurses involved in the care pathway and through patient feedback. From February 2018 (DH and KH), use of triclosancoated sutures was done in all tissue layers excluding the skin. From September 2018 (DH) and April 2019 (KH), all patients, from the evening before surgery and until hospital discharge (alternatively fifth postoperative day), followed a two times per day standardised operating procedure for decolonisation consisting of nasal decontamination by mupirocin $2 \%$ nasal ointment, universal skin decontamination by chlorhexidine gluconate washcloths and oral decontamination by chlorhexidine mouthwash. All subjects were thoroughly instructed in the decolonising procedure and remedies supplied free of charge. Patients admitted for planned surgery performed all pre-surgery intervention at home. A low number of patients, with presumed low compliance to the decolonising procedure, were admitted the day before surgery. From time of admission, all patients were supervised and supported according to need, to ensure good compliance. Patients reporting allergy towards chlorhexidine or developing subjective signs of skin irritation discontinued use of chlorhexidine, but continued the use of mupirocin.

For all patients, chlorhexidine alcohol was used for surgical skin preparation.

The supervising nurse used a checklist for assessment of compliance to procedures.

Results for all cases from 2012 onwards were plotted in p-chart including binomial confidence intervals using LifeQI (Life QI System, UK), a web-based healthcare quality improvement tool. 


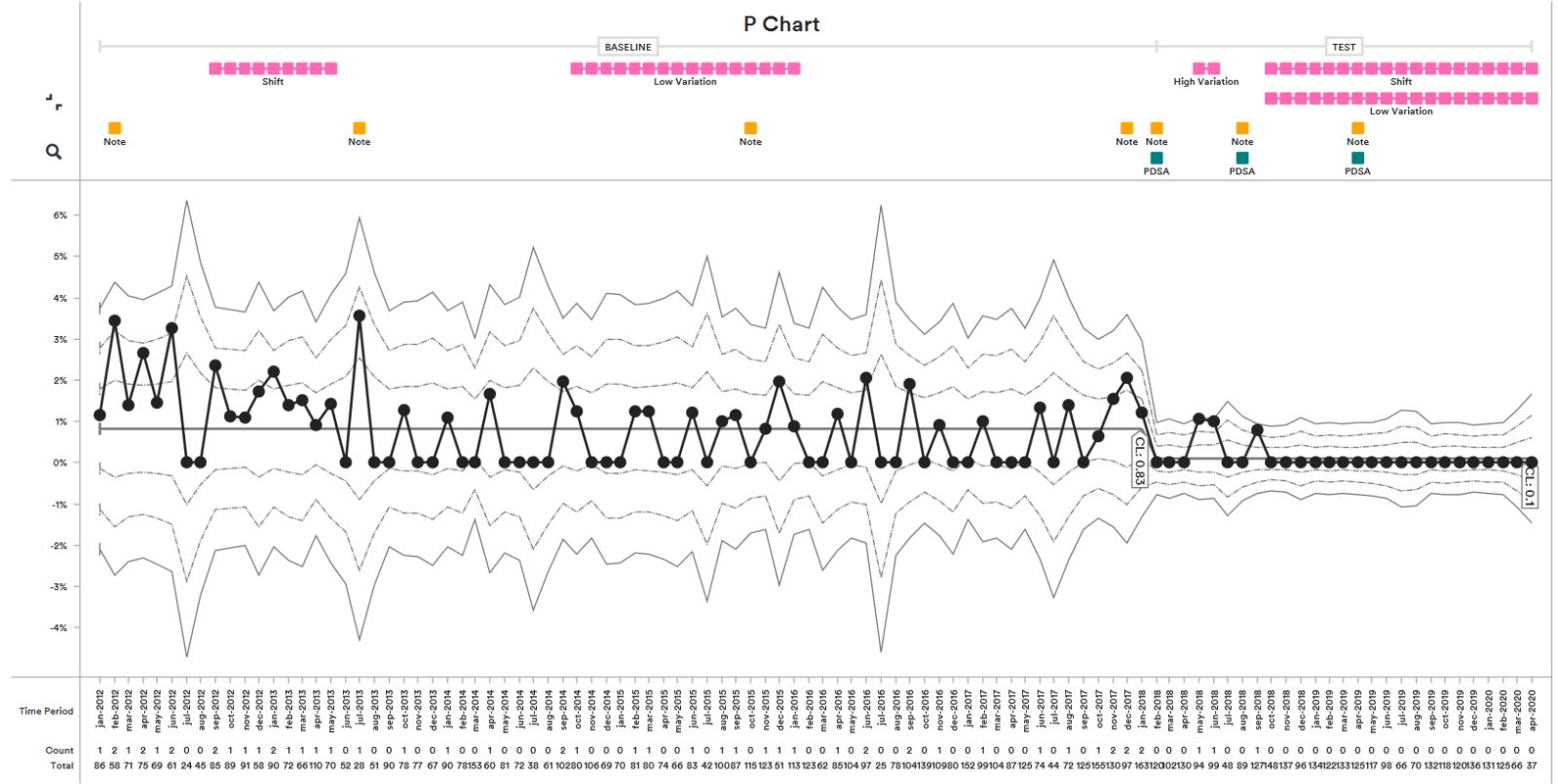

Figure 1 P-chart showing monthly incidence of prosthetic joint infections caused by Staphylococcus aureus. Outlier-a single point above the +3 sigma or below -3 sigma limit. Shift - a run of eight points in a row above or below the centerline. Note, a point on the centerline neither adds nor resets the run but is ignored, that is, if you have seven points above the centerline and the eighth on the centerline then the trigger is dependent on the ninth point. If it is above, it makes the run of eight; if it is below, then it halts the run. Trend-six consecutive points decreasing or increasing. Note, as with the shift rule, a point equal to the last point does not add to nor resets the trend. High variation - two out of three points in the outer one-third of the chart, between the +2 and +3 sigma or between the -2 and -3 sigma limits. Note, a point on the \pm 2 sigma limit does not count as in the outer third. Low variation -15 consecutive points in the inner third of the chart, between the -1 and +1 sigma limits. Note, a point on the limit does not count. PDSA, Plan-Do-Study-Act.

PJI cases were defined using criteria from the Center for Disease Control's criteria, ${ }^{8}$ based on systematic chart review including infection surveillance forms and microbiological data from cultures of deep tissue samples.

This quality improvement project had no external funding.

\section{RESULTS}

From 2012 to March 2020, n=9048 patients underwent surgeries with a prosthesis implant (1430 acute, 7618 elective) (6778 hip, 1968 knee and 302 shoulder). There was no significant change in the proportion of the different surgical procedures over time. $\mathrm{DH}$ and $\mathrm{KH}$ get a large proportion of hip replacement cases from other trusts, which explains the skewed distribution.

The incidence of PJI with $S$. aureus was $0.8 \%(0.6 \%-1.0 \%$; $\mathrm{n}=54)$ before and $0 \%(0.0 \%-0.2 \%$; $=0)$ after the implementation of the combined intervention. The last case recorded was in September $2018(\mathrm{KH})$. After this last case, 2216 patients had surgery with prosthesis implantation, without any recorded PJI with $S$. aureus. The p-chart shows a level shift and low variation from October 2018 (figure 1).

\section{DISCUSSION}

Our quality improvement project has reduced the incidence of deep prosthesis infections with $S$. aureus to zero. The strength of the project is that we have implemented changes with solid scientific documentation, with local process optimisation that ensured high compliance and control over the processes. We have found the impact to be highly robust over time-even in daily routine practice. The fact that the protocol also ensures compliance for resource-poor patients and includes non-elective cases is a major strength. With a direct cost of less than $€ 50$ per surgery, the project is highly cost-efficient. However, our hospital organisation has a high degree of standardisation in the perioperative processes. Whether one can achieve equally good results in hospital organisations with less process standardisation is uncertain. We would recommend other hospitals to consider using our protocol. We are now in a sustain phase regarding $S$. aureus $\mathrm{PJI}$, and we continue to expand our quality improvement efforts to reduce the corresponding incidence of PJI for non-S. aureus which remained largely unchanged at $0.4 \%$ throughout the study. It is also important that we become significantly better at preventing these infections on our journey to zero serious PJIs.

Contributors These authors contributed equally: ED, MW, VH-H, MO, SV and LM.

Funding The authors have not declared a specific grant for this research from any funding agency in the public, commercial or not-for-profit sectors.

Competing interests None declared.

Patient and public involvement Patients and/or the public were involved in the design, or conduct, or reporting, or dissemination plans of this research. Refer to the Methods section for further details.

Patient consent for publication Not required. 
Ethics approval The project was a healthcare improvement project that did not need ethical approval according to Norwegian law.

Provenance and peer review Not commissioned; externally peer reviewed.

Open access This is an open access article distributed in accordance with the Creative Commons Attribution Non Commercial (CC BY-NC 4.0) license, which permits others to distribute, remix, adapt, build upon this work non-commercially, and license their derivative works on different terms, provided the original work is properly cited, appropriate credit is given, any changes made indicated, and the use is non-commercial. See: http://creativecommons.org/licenses/by-nc/4.0/.

\section{ORCID iD}

Lukas Maansson http://orcid.org/0000-0002-3954-5839

\section{REFERENCES}

1 Parisi TJ, Konopka JF, Bedair HS. What is the long-term economic societal effect of periprosthetic infections after THA? A Markov analysis. Clin Orthop Relat Res 2017;475:1891-900.
2 National Institute for Health and Care Excellence (NICE). Surgical site infections: prevention and treatment. London, 2020.

3 Tang J, Hui J, Ma J, et al. Nasal decolonization of Staphylococcus aureus and the risk of surgical site infection after surgery: a metaanalysis. Ann Clin Microbiol Antimicrob 2020;19:33.

4 WHO Guidelines Approved by the Guidelines Review Committee. Global guidelines for the prevention of surgical site infection. 2018. Geneva: World Health Organization, 2018.

5 Berríos-Torres SI, Umscheid CA, Bratzler DW, et al. Centers for disease control and prevention guideline for the prevention of surgical site infection, 2017. JAMA Surg 2017;152:784-91.

6 Barbero JM, Romanyk J, Vallés A, et al. [Decolonization for Staphylococcus aureus carriers in arthroplasty surgery after hip fracture]. Rev Esp Quimioter 2017;30:264-8.

7 Manian FA. The role of postoperative factors in surgical site infections: time to take notice. Clin Infect Dis 2014;59:1272-6.

8 Horan TC, Andrus M, Dudeck MA. CDC/NHSN surveillance definition of health care-associated infection and criteria for specific types of infections in the acute care setting. Am J Infect Control 2008;36:309-32. 